\title{
Les carrières de cadres au cœur des restructurations : la recomposition des effets de genre? \\ L'internationalisation d'un groupe français en Angleterre et en Hongrie
}

\author{
Cécile Guillaume, Sophie Pochic
}

\begin{abstract}
Résumé
L'effet des restructurations sur les carrières des cadres reste encore peu analysé, notamment dans une perspective genrée. Les deux cas de fusion-acquisition étudiés, en Angleterre et en Hongrie, montrent que si la recomposition des espaces d'opportunité semble en partie favorable aux carrières féminines, le renforcement d'un modèle d'activité masculin-neutre contribue au durcissement du plafond de verre, pour tous ceux qui ne peuvent se conformer à ces attendus, en particulier les femmes.
\end{abstract}

Mots Clé : restructuration, fusion-acquisition, plafond de verre, genre, cadres, carrière, changement organisationnel, internationalisation

Alors que la sociologie s'est intéressée de façon régulière au chômage depuis les années quatre-vingt, de façon paradoxale, elle ne l'a abordé que récemment sous l'angle des fusions-acquisitions et des restructurations d'entreprise, des victimes et/ou survivants de plans sociaux (Guyonvarch, 2007; Linhart, 2002 ; Clot, 1994) ${ }^{1}$. Ceux qui gèrent ces réorganisations et parfois font carrière dans et sur ces restructurations, les cadres, sont souvent l'angle mort de ces enquêtes, à part de rares exceptions (Raveyre, 2005; Beaujolin, 1999; Dejours, 1998). Du côté anglo-saxon, davantage de travaux se sont penchés sur l'analyse des effets des réorganisations sur les carrières des cadres, mais ils intègrent rarement les dimensions de genre dans l'analyse. Pourtant la question se pose. Les fusionsacquisitions ont-elles des effets différenciés sur les carrières des hommes et des femmes ? Dans un contexte de forte féminisation de l'encadrement, les femmes ont-elles bénéficié ou au contraire pâti de la déstructuration des marchés internes du travail et de leur recomposition à une échelle internationale ?

Des recherches récentes soulignent les risques de renforcement de la ségrégation professionnelle et la fragilisation des carrières des femmes cadres dans des contextes organisationnels mouvants (Stevens, 2007 ; Collins, 2005 ; Rasmussen, 2001 ; Tienari, 2000 ; Karambayya, 1998). Mais le nombre de travaux empiriques sur ce thème reste faible et n'intègrent que très rarement les effets des contextes sectoriels et nationaux. Nous avons demandé à un grand groupe français du secteur de l'énergie, au sein duquel nous avions déjà mené une enquête en 2004 sur les carrières des cadres dirigeants (Guillaume, Pochic, 2007), de nous ouvrir les portes de deux de ses filiales, l'une anglaise et l'autre hongroise (cf. encadré méthodologique). Nous montrerons dans un premier temps comment le contexte de contraction des marchés du travail à l'échelle locale (les filiales) et de tertiarisation de l'activité liée à la dérégulation du secteur énergétique permet

\footnotetext{
${ }^{1}$ Intérêt sans doute à replacer dans la forte augmentation des fusions-restructurations en France à la fin des années 1990, dont les effets sur l'emploi sont cependant contrastés (Bunel et al., 2007).
} 
d'éclairer le caractère sélectif et plafonné du processus de féminisation. Une part croissante de femmes ne signifie cependant pas automatiquement une amélioration de leurs perspectives de carrière. Dans ces contextes d'instabilité organisationnelle forte et de lutte des places, se renforce en filiale un modèle d'activité masculin-neutre, combinant exigences de mobilité géographique contrainte et extension des horaires de travail, que nous décrypterons ensuite. Dans une dernière partie, nous évoquerons comment la recomposition des mécanismes de cooptation à une autre échelle favorise les carrières des hommes dotés des bons atouts de départ. Nous conclurons sur les risques de plafonnement de carrière, voire d'exclusion, pour tous ceux qui ne peuvent se conformer aux nouvelles exigences de l'entreprise, et notamment les femmes, dans un contexte de très forte asymétrie entre les filiales et la maison-mère.

\section{Méthodologie : enquête croisée sur deux filiales d'un groupe français}

L'histoire interne du processus d'internationalisation du Groupe, dont un tiers du personnel est situé désormais à l'étranger, a tout d'abord été retracé par des interviews de cadres responsables ou acteurs de cette politique de fusions-acquisitions dans différentes directions de la maison-mère à Paris (20 cadres, dont 6 étrangers), complétés par l'étude de documents d'entreprise. Nous avons ensuite réalisé en 2006 des interviews dans deux filiales européennes, avec les cadres français expatriés (à des postes de direction de la filiale, 3 hommes en Angleterre et 2 hommes en Hongrie) et les « cadres locaux » dans chaque filiale à différents niveaux de la hiérarchie (18 en Angleterre dont 7 femmes, et 18 en Hongrie dont 7 femmes). Nous sommes retournés en 2008 revisiter ces filiales et faire des entretiens complémentaires auprès de cadres et de techniciens (10 en Angleterre, dont 4 femmes et 12 en Hongrie, dont 5 femmes).

En sachant que la catégorie des cadres en France est beaucoup plus institutionnalisée que partout ailleurs, nous avons sélectionné des «équivalents fonctionnels » dans ces filiales (à partir de la fonction occupée, du salaire et des responsabilités indiquées dans l'organigramme), afin de saisir localement les différences sociétales de production de la hiérarchie en entreprise. Nous eu accès à des statistiques sexuées sur le personnel à partir d'une extraction anonymisée du fichier du personnel de 2006 dans la filiale anglaise et de tableaux réalisés «à façon » dans la filiale hongroise en 2008 (cf. tableaux 3 et 4 en annexe). Les Labour Force Surveys de $2005^{2}$ permettent de définir à grands traits les spécificités de l'emploi des salariés qualifiés de chaque pays et du secteur Energie et eau (cf. tableaux 1 et 2).

La méthode des « récits de vie» (Bertaux, 1996) vise à articuler parcours professionnel et vie privée, mais aussi à ancrer ces parcours dans l'histoire de la filiale, dans le marché du travail local, dans le contexte sectoriel et national (valeur des diplômes, politiques économiques et sociales). Quatre portraits de carrière sont développés dans le texte afin de retracer les séquences, les «turning points» et les autruis significatifs de la carrière dans une tradition interactionniste (Glaser, 1968). Les entretiens en Angleterre ont été réalisés et retranscrits en anglais. Sauf pour quelques salariés bilingues, la majorité des entretiens en Hongrie ont été réalisés avec un interprète, avec une traduction en consécutif et retranscrits directement en français. Le fait d'être accompagné par un interprète professionnel n'a pas surpris les salariés hongrois, puisque les cadres français expatriés travaillent au quotidien avec un interprète à leurs côtés. Toutefois pour contrôler d'éventuels biais liés à cette médiation, toutes les retranscriptions ont été relues par une lectrice de hongrois, ce qui a permis de confirmer le grand respect des paroles aussi bien de l'intervieweur que des interviewés. Nous voulons remercier ici Csaba Gáldi, Eva Gáldi et Mónika Takács pour leur précieuse collaboration.

\footnotetext{
${ }^{2}$ Nous remercions le réseau Equalsoc, et plus particulièrement Benoit Tudoux, pour l'accès à des bases de données. L'utilisation de nomenclatures harmonisées comme «Managers and professionals » ou les niveaux de qualification ISCED posent évidemment des problèmes de comparabilité liés à l'harmonisation, que nous n'évoquerons pas ici faute de place.
} 


\section{L’internationalisation du groupe : une opportunité pour les femmes ?}

Les fusions-acquisitions ont souvent en pratique comme conséquence des restructurations et des suppressions d'emplois, parfois à moyen terme et en vagues successives, surtout pour les emplois peu qualifiés (Lefresne, Sauviat, Grimault, 2008). On connaît par contre moins leurs effets sur la structure des emplois qualifiés, dans des contextes complexes pouvant combiner une forte pression à la rationalisation (et une baisse d'effectifs), mais également des recrutements de jeunes cadres sur des spécialités tertiaires contribuant à une féminisation sélective mais plafonnée.

\subsection{La « tentation internationale » d'un groupe français}

L'entreprise étudiée est passée en quinze ans d'une configuration de monopole public présent uniquement en métropole et dans les DOM-TOM à un groupe de taille internationale implanté dans trente pays qui réalise $39 \%$ de son chiffre d'affaires à l'étranger en 2006 (sur un total de 27 milliards) et qui évalue à 32\% ses salariés hors de France en 2006 (sur un total de 50.000 salariés $^{3}$ ), ce qui en fait un des acteurs énergétiques leader en Europe. Cette ouverture à l'international s'est réalisée sans véritable stratégie concertée, de façon très pragmatique et a été portée par quelques acteurs-clés dans certaines directions. Du côté du transport et de la distribution de gaz (métiers historiques de l'entreprise), la chute du mur de Berlin a ouvert la possibilité d'acquérir intégralement ou via des prises de participation les infrastructures d'anciennes entreprises publiques (la Hongrie dès 1995) afin de sécuriser l'approvisionnement en gaz naturel, notamment avec le principal fournisseur, le russe Gazprom ${ }^{4}$. L'entreprise a également depuis 2000 à la fois investi dans des licences d'exploration et de production de gaz naturel dans dix pays, mais aussi développé des activités de commercialisation et de « trading » de gaz (achat à court et long terme), en rachetant parfois des entités déjà existantes comme en Angleterre. Enfin pour diversifier ses activités et fidéliser ses clients, l'entreprise a développé une offre de services (gestion des déchets, nettoyage industriel, climatisation/chauffage) via l'acquisition de filiales françaises et européennes. Cette internationalisation encore récente fondée sur une croissance externe pourrait être caractérisée comme la «tentation internationale » (Lorrain, 2005) d'un groupe qui demeure encore très franco-français dans ses centres de pouvoir, ses réseaux d'information, ses procédures de prises de décision.

Cette stratégie s'inscrit dans un marché énergétique européen en voie de libéralisation, où chaque pays est plus ou moins avancé dans la dérégulation et l'ouverture à la concurrence étrangère (avec des étapes progressives en fonction de la taille des clients, des professionnels aux particuliers). Cette dérégulation amène notamment à séparer juridiquement l'activité de transport, de stockage d'énergie et de distribution sur un territoire (monopole «naturel») de sa production ou de sa vente aux clients, afin que des concurrents puissent accéder aux infrastructures. L'Angleterre est en avance sur cette dimension puisque le gouvernement a libéralisé le marché du gaz entre 1986 et 1996 et le marché de

\footnotetext{
${ }^{3}$ Le nombre de salariés est « consolidé » c'est-à-dire représente la masse salariale en équivalent temps plein.

${ }^{4}$ Principal fournisseur de l'entreprise, avec lequel l'entreprise a des relations de partenariat depuis 1975, avec parfois des filiales communes.
} 
l'électricité entre 1990 et 2000. La France et la Hongrie suivent le même calendrier fixé en 1998 par la DG «Transport, Energie » de la Commission européenne pour instaurer le marché unique de l'énergie: ouverture aux professionnels depuis 2004 et ouverture aux particuliers théoriquement depuis 2007. Mais si la France possède encore deux entreprises publiques EDF et Gaz de France ${ }^{5}$, le secteur de l'énergie a été complètement privatisé en Hongrie depuis 1995. La maison-mère du groupe étudié fonctionne donc encore avec un marché interne du travail presque fermé sur le territoire français avec un statut très protecteur (Guillaume, Pochic, 2007), alors qu'elle participe activement aux restructurations du secteur dans des pays étrangers en tant qu'actionnaire.

\subsection{De fortes turbulences sur les marchés internes}

Avant les grandes vagues de privatisation des années 90, le secteur de l'énergie en Angleterre et en Hongrie était comme en France caractérisé par des entreprises publiques de grande taille sur un marché de monopole. Ces entreprises pouvaient couvrir un territoire national (comme British Gas ou Mól en Hongrie) ou seulement une région (Yorkshire Electricity ou Égáz sur la région du Nord Ouest de la Hongrie). Les cadres - hommes ou femmes, ingénieurs et autres - entrés dans ces grandes entreprises $\mathrm{y}$ ont donc connu des «carrières bureaucratiques/organisationnelles » caractéristiques des marchés internes du travail (Glaser, 1968 ; Doeringer, Piore, 1971). Avec la privatisation et l'ouverture à la concurrence de ces anciens monopoles, ces marchés internes de l'emploi ont traversé depuis dix ans de fortes turbulences liées à des fusions-acquisitions répétées avec souvent scission d'une partie de l'activité, parfois revendue, parfois filialisée. Ces rachats, souvent par des actionnaires étrangers, ont tous été suivis par des processus de réorganisation qui ont diminué la taille de l'entreprise et réduit les échelons hiérarchiques. Les marchés internes se sont donc à la fois ouverts (davantage de points d'entrée et de sortie) et contractés (moins de postes à pourvoir en interne), mais selon des processus différents suivant chaque filiale. Ces périodes de réorganisation ont été en même temps l'occasion de renouvellement des cadres, via des recrutements, pour accompagner la dérégulation du marché de l'énergie.

Le processus de réduction des effectifs peut être retracé en continu en Hongrie puisque la filiale est issue de la fusion des deux entreprises régionales (DEGAZ au Sud-Est et EGAZ au Nord-Ouest) rachetées par un seul actionnaire depuis leur privatisation en 1995. Les effectifs sont passés de 2.400 salariés lors du rachat à 1.200 en 2006 lors de l'enquête ${ }^{6}$ et ont continué de baisser, pour atteindre 1.050 salariés en 2008. Les statistiques annuelles transmises au Ministère (cf. tableau 3) reflètent la progressive externalisation de nombreux services techniques (relevé des compteurs, dessin des réseaux de gaz), les emplois de "cols bleus ${ }^{7}$ (des ouvriers et ouvrières peu qualifiés jusqu'aux techniciens et agents de maîtrise) ayant fait l'objet de vagues successives de réduction, passant de 1.100 à un peu plus de 300 salariés. Les cadres n’ont pas été épargnés par la diminution du

\footnotetext{
${ }^{5}$ Même si la fusion avec Suez et donc la privatisation de cette dernière a été actée en septembre 2007.

${ }^{6}$ Les départs de l'entreprise sont supérieurs à 1.200 car sur cette période l'entreprise a également recruté, surtout pour des postes qualifiés.

${ }^{7}$ Les statistiques officielles ne permettent de distinguer que les « cols bleus » des « cols blancs » et ne permettent pas d'avoir un niveau plus fin sur le niveau de qualification des postes/individus.
} 
nombre de services techniques et d'agences, mais ont souvent été protégés du risque de chômage soit par leur âge (les plus âgés ayant pu partir via des préretraites ou des congés maladie), soit par leur diplôme et leurs réseaux ${ }^{8}$. Cette filiale confirme donc ce que montre au niveau national la Labour Force Survey de 2005 (cf. tableaux 1a et $1 \mathrm{~b}$ ) : grâce à leur niveau élevé de diplôme, les cadres hongrois ont traversé sans encombres, voire ont bénéficié pour certains, de l'arrivée des actionnaires étrangers ${ }^{9}$. La gestion des restructurations par cette filiale est considérée par les salariés hongrois comme relativement « sociale », à la fois grâce aux cadres locaux (et notamment le PDG) qui ont continuellement tempéré les velléités des expatriés venus pour "faire du chiffre ", d'une convention collective relativement protectrice (niveau d'indemnités élevé, même avec un syndicat peu offensif), mais surtout en comparaison de l'attitude d'autres entreprises étrangères en Hongrie. Malgré ces coupes sombres, la filiale peine cependant à atteindre les objectifs fixés par l'actionnaire car le gouvernement social-libéral maintient le prix du gaz en dessous du prix du marché, dans un pays durement touché par la transition vers l'économie de marché ${ }^{10}$.

Cette réorganisation s'est accompagnée d'un renouvellement des cadres, plutôt sur des profils d'experts, parfois sur des métiers déjà existants dont le rôle est renforcé (comme les Ressources Humaines) ou sur de nouveaux métiers en croissance (liés à la captation et la fidélisation de la clientèle ou aux nouvelles normes juridiques encadrant les contrats d'achat et de vente). Pour assumer ces nouvelles fonctions, les expatriés français ont favorisé le recrutement par annonce de jeunes diplômés bilingues, considérés comme plus ouverts à la libéralisation (que les anciens cadres marqués par leur expérience d'une entreprise d'Etat monopolistique) mais permettant aussi de limiter la masse salariale. L'entrée de cadres expérimentés aux salaires élevés en position d'encadrement a été très limitée, ciblée sur des cadres venant d'entreprises privées étrangères, mais rarement débauchés des concurrents du secteur. Ces mouvements d'entrée et de sortie expliquent que la moyenne d'âge ait progressivement baissé, pour atteindre 42 ans en 2008, alors que la stabilité de l'emploi reste forte, avec une ancienneté moyenne de 14 ans (cf. tableau 5).

Ce processus de réduction des échelons hiérarchiques et de la taille de l'entreprise est plus complexe à retracer pour la filiale anglaise, créée en 2002, car celle-ci est révélatrice des acquisitions répétées qu'a connu le secteur énergétique anglais depuis les années $1990^{11}$. Cette filiale de la taille d'une PME (190 salariés) s'occupe uniquement de commercialisation d'énergie, soit d'achat et de vente, notamment à destination de clients de grande taille (entreprises ou collectivités), et ne gère aucune infrastructure technique ${ }^{12}$. Elle est majoritairement composée de cols blancs, surtout de "managers and professionals », relativement jeunes, 33

\footnotetext{
${ }^{8}$ Les chefs de centre s'étant préoccupés de retrouver un poste pour leurs fidèles collaborateurs, parfois au prix d'une mobilité latérale, d'un déclassement et/ou d'un déménagement.

${ }^{9}$ A l'inverse les peu diplômés, et notamment les femmes, ont été fortement touchés à la fois par des périodes longues de chômage, voire par un retrait du marché du travail officiel (leur taux de chômage est à $14 \%$ avec un taux d'emploi inférieur à $30 \%$ ).

${ }^{10}$ Les chaudières collectives défectueuses de nombreux immeubles génèrent ainsi des factures très élevées, sans que les mairies ou les particuliers aient les moyens de les rénover.

${ }^{11}$ Les statistiques du personnel ne permettent ainsi de remonter qu'à 2002, date de sa « création ».

12 Elle possède une seule centrale électrique dont le personnel d'exploitation est externalisé dans une autre filiale du Groupe.
} 
ans en moyenne et recrutés depuis moins de 2 ans pour $40 \%$ d'entre eux (cf. tableau 4). Malgré son chiffre d'affaire élevé (le deuxième des filiales du Groupe), sa rentabilité reste subordonnée à la volatilité des cours de l'électricité et du gaz dans un marché très concurrentiel (" un marché coupe-gorge » selon un expatrié) et sa revente est parfois évoquée.

Le noyau dur de cette filiale est constitué d'une quarantaine de cadres, seniors et de trentenaires, venant pour partie d'une «spin-off ${ }^{13}$ » de l'ancienne entreprise publique régionale (précisément, le service Trading de Yorkshire Electricity ${ }^{14}$, basée principalement à Leeds). Privatisée en 1990, cette entreprise est vendue à un groupe américain en 1997, puis revendue à un groupe anglais en 2001 (une ancienne entreprise publique elle aussi). La "réorganisation» qui suit cette deuxième acquisition, la séparation de l'activité de distribution et d'achat d'énergie avec relocalisation à $150 \mathrm{~km}$ (Swindon), s'accompagne de nombreux licenciements « volontaires $»{ }^{15}$ ou pour motif personnel, notamment pour refus d'une autre offre (refus de déménagement ou de déclassement) ${ }^{16}$. Sur 3000 salariés, seuls 700 ont eu une proposition de ré-embauche et seuls 12 l'ont finalement accepté. C'est à cette occasion qu'un petit groupe de "senior managers " se lance dans la création d'une entreprise de commercialisation d'énergie à Leeds, avec le soutien financier d'un groupe allemand dont ils deviennent une filiale. Ils proposent à 30 "personnes-clés» de les suivre, majoritairement des jeunes cadres qui travaillent sous leurs ordres. Le rachat surprise six mois après par un groupe allemand de l'entreprise anglaise qu'ils viennent juste de quitter (ce qui signifie à terme la fermeture de leur bureau à Leeds et la relocalisation à Swindon) les oblige à demander à être mis en vente. Le groupe français qui les rachète en 2002 les fusionne aussitôt avec deux autres de ses filiales, mais en conservant le site de Leeds comme plateforme.

Autour de ce noyau dur se sont agrégés majoritairement des jeunes cadres, recrutés directement après leur diplôme universitaire (degree ou bac +3 ) ou après une première expérience professionnelle (en cabinet d'audit notamment). Les cadres seniors recrutés en cours de carrière, souvent via des chasseurs de tête, sont peu nombreux, mais davantage présents qu'en Hongrie. Certains ont circulé entre différents opérateurs du secteur, leur mobilité étant parfois à leur initiative (insatisfaction sur le salaire, leur carrière ou le style de management) mais aussi liée à l'instabilité du marché de l'énergie (fusions, restructurations et relocalisations). Après un an de tension, l'équipe dirigeante de l'autre filiale située à Telford $(200 \mathrm{~km})$ a d'ailleurs quasiment disparu, seul un dirigeant ayant accepté de rester après la relocalisation sur Leeds (moyen choisi par l'actionnaire pour résoudre les conflits de pouvoir). Ceci est en accord avec la plus grande mobilité

\footnotetext{
${ }^{13}$ Spin off ou demerger : action inverse d'une fusion, quand une entreprise se sépare d'un pan de son activité qui devient une entreprise juridiquement autonome.

${ }^{14}$ En 1982, Yorkshire Electricity avait ainsi 7.944 salariés dont 69 managerial staff (Source: Yorkshire Electricity Board).

15 Cette technique du «voluntary redundancy» a été souvent utilisée en Angleterre pour restructurer les entreprises publiques et l'administration, l'employeur demandant des « volontaires » en échange d'un dédommagement financier.

${ }^{16}$ British Gas a ainsi diminué son personnel de moitié entre 1995 et 1997 sans plan social mais via une « réorganisation": passant de 12 régions à 5 business units, tous les salariés devaient repostuler sur des offres d'emploi réduites de moitié, certains ayant accepté de troquer déclassement contre stabilité...
} 
externe des salariés qualifiés anglais mesurée par la Labour Force Survey de 2005 , avec plus qu'un quart des " managers and professionals » recrutés depuis moins de 5 ans, que ce soit au niveau national ou au niveau du secteur de l'énergie, contre 18\% en Hongrie et 9\% en France (cf. tableau 1b).

Ces moments de recomposition du marché de l'énergie (arrivée de nouveaux acteurs de petite taille avant une probable consolidation autour de quelques grandes entreprises multinationales selon les experts) ne signifie cependant pas la fin des marchés internes et des perspectives de carrière pour les cadres. Ils amplifient les inégalités de carrière au sein des cadres travaillant dans ce secteur, suivant leur âge et leur position professionnelle, à l'image en France des accidents de carrière des cadres (Pochic, 2009) ou des licenciements de cadres pour motif personnel (Palpacuer, Seignour, Vercher, 2007). Tout en valorisant la mobilité externe, surtout pour ceux qu'elles ne souhaitent pas garder, les entreprises cherchent à fidéliser les cadres à potentiel pour ne pas perdre des compétences rares (Marsden, 1989), mais aussi parfois des clients ou des informations stratégiques. Les jeunes cadres recrutés aux premiers niveaux de responsabilités et d'expertise espèrent tous pouvoir être récompensés de leur investissement au travail $^{17}$ et de leurs résultats par des promotions internes ${ }^{18}$. Le faible nombre d'échelons hiérarchiques, le strict contrôle de la masse salariale et la tension sur le marché local du travail des jeunes cadres ${ }^{19}$, surtout dans les spécialités financières et commerciales ${ }^{20}$, explique en partie le fort turn-over observé dans les premiers niveaux de cadres. Les dirigeants des deux filiales sont d'ailleurs préoccupés par ce turn-over, et essayent de le compenser par la mise en place d'une politique de détection des cadres à potentiel et d'offre de formations (notamment en langues). Du côté des cadres d'âge intermédiaire, la taille réduite de l'entreprise diminue leurs opportunités de progression de carrière, que ce soit verticalement ou même latéralement, et seul le départ de certains peut libérer des postes. L'arrivée d'un expatrié ou d'un senior débauché d'un concurrent rend en plus difficile la projection de carrière dans un climat de forte incertitude sur l'avenir de l'entreprise. Parmi les plus âgés, les plus dotés en ressources peuvent encore envisager la mobilité externe (consultant par exemple), alors que d'autres ont modéré leurs ambitions (notamment pour préserver leur équilibre familial), voire ont des objectifs plus « prosaïques » (conserver son poste et son salaire).

\subsection{Une féminisation sélective et plafonnée}

Ces mouvements de sorties et d'entrée de main d'œuvre ont profondément modifié le profil des cadres des deux filiales: davantage sur des spécialités financières et commerciales, plus jeunes, plus diplômés et surtout plus féminisés. Avec $33 \%$ de femmes dans la filiale anglaise et même $41 \%$ dans la filiale

\footnotetext{
${ }^{17}$ Les anglais utilisent la notion de « commitment » pour désigner cet investissement.

18 Malgré les discours gestionnaires sur la progression des «carrières nomades » (Boltanski, Chiapello, 1999 ; Bender et al., 2003), les marchés internes sont toujours attractifs, comme l'ont montré des enquêtes sociologiques sur les cadres en Australie dans les grandes entreprises (Martin et al., 2000) ou en Angleterre dans le secteur de la finance (Devine et al., 2000).

19 En Hongrie, le site de Szeged, région peu industrialisée avec un fort taux de chômage est beaucoup moins concerné par ce problème que le site de Györ, région très industrialisée avec un faible taux de chômage, et encore moins que Budapest.

${ }^{20}$ En Angleterre, Leeds est la deuxième place financière après Londres.
} 
hongroise ${ }^{21}$, on dépasse dans ces petites structures les taux de féminisation du secteur énergétique obtenus par les Labour Force Surveys (19,5\% pour l'Angleterre et $22 \%$ pour la Hongrie pour les " managers and professionals 》 du secteur énergie et eau, taux qui monte même respectivement à 30\% et $39 \%$ quand on se restreint aux moins de 35 ans, cf. tableau $1 b$ ). En raison de la ségrégation sexuée des filières de formation, que l'on retrouve en Hongrie comme en Angleterre, les femmes sont en effet davantage présentes dans les viviers du recrutement actuel de ces deux filiales. Celles-ci recherchent en priorité des diplômés avec des compétences en commerce, finance ou droit, que ce soit après l'obtention de leur diplôme pour des postes de « junior » ou après une expérience professionnelle dans le privé pour des postes de «senior». Les femmes sont ainsi $45 \%$ dans la filiale anglaise dans les postes d'entrée pour les jeunes diplômés (credit control, finance accountant, sales support et le niveau juste au dessus de « team leader »), cf. tableau 3. Elles sont de même dans la filiale hongroise $88 \%$ chez les experts administratifs ( $\mathrm{RH}$, communication), 38\% chez les experts commerciaux et financiers, mais seulement $20 \%$ sur les postes d'experts techniques (postes d'entrée des ingénieurs diplômés, mais accessibles également via la promotion interne des techniciens), cf. tableau 5.

La minoration progressive du poids des ingénieurs au profit de profils plus «business» a donc objectivement ouvert des opportunités aux femmes, alors même qu'elles étaient relativement invisibles antérieurement dans un monde dominé par les ingénieurs. Dans ces deux pays, les ingénieurs diplômés (majoritairement des hommes) étaient auparavant les mieux placés pour atteindre les postes de direction dans ces industries avec des infrastructures techniques et risquées (puits, tuyaux, stockage de gaz par exemple) et des cols bleus qualifiés (majoritairement des hommes) en nombre élevé. En Hongrie sous le régime communiste, le capital politique ${ }^{22}$ était au moins aussi essentiel que le capital scolaire, ce qui constituait un obstacle aux carrières des femmes, qui étaient considérées comme «moins fiables politiquement» car moins disponibles en raison d'une division des tâches domestiques très traditionnelle (Fodor, 2002). Avec l'ouverture de la Hongrie à l'économie de marché dès 1968, puis la promotion de la «seconde économie ${ }^{23}$ dans les années 1980, les entreprises commencent cependant à être moins regardantes sur le capital politique et beaucoup plus sur l'expertise et les diplômes (Fodor, 2003). Cette tendance conjuguée à une politique égalitariste d'accès aux études supérieures ${ }^{24}$ et de soutien de l'activité féminine à temps plein explique que les femmes soient plus présentes en moyenne parmi les «managers and professionnals» en Hongrie,

\footnotetext{
${ }^{21}$ Dans cette réduction d'effectifs, le taux de féminisation ne s'est que légèrement amélioré, passant de $38 \%$ en 1996 à $41 \%$ en 2007 , car les ouvrières ont été particulièrement touchées par les réductions des effectifs : des 228 femmes cols bleus de 1996, il n'en reste que 4 dix ans après...

${ }^{22}$ Capital politique à différentes dimensions : ne pas venir d'une famille stigmatisée par un passage à l'Ouest ou par ses origines (bourgeoises ou indépendantes) ; être considéré comme un bon communiste (par son engagement actif dans les mouvements de jeunes ou le syndicat ou encore mieux le Parti) et si possible être soutenu par les réseaux du Parti, notamment dans des industries stratégiques comme l'énergie.

${ }^{23}$ Soutien à la création des petites entreprises personnelles (GMK / Gazdasági munkaközösség / communauté du travail à but lucratif) sur le lieu du travail avec les salariés de la même usine, travaillant souvent comme sous-traitants de l'entreprise, le soir ou le week-end.

${ }^{24}$ En terme de niveau (via des quotas), mais pas en terme de spécialités, les femmes étant minoritaires dans les filières techniques en raison de processus genrés d'orientation scolaire.
} 
$49 \%$ tous secteurs confondus contre $39 \%$ en Angleterre ${ }^{25}$. De manière conforme, les contremaîtres et même les chefs de département, d'agences ou de filiales sont composés d'un tiers de femmes dans la filiale hongroise (tableau 4), alors que les cadres et experts ("managers and analysts») comportent moins d'un quart de femmes dans la filiale anglaise (tableau 3). Malgré ces différences d'héritage, dans les deux pays, quelques femmes diplômées trentenaires considèrent que les restructurations ont été bénéfiques pour elles, car elles ont créé ou libéré des postes sur lesquels elles ont pu connaître des accélérations de carrière, à l'image d'Anna.

\section{Anna ou les opportunités pour les commerciales}

A 34 ans, Anna est cadre commercial («sales manager ») dans la filiale anglaise et gagne un salaire de $36485 £$ annuel pour un temps partiel de $30 \mathrm{~h}$ sur 4 jours. Ne voulant pas aller à l'université après son bac scientifique, elle obtient en 1990 l'opportunité de faire une formation en apprentissage $^{26}$ en management chez British Gas, grâce à son père ingénieur promu qui avait commencé sa carrière dans cette entreprise. Quand elle est diplômée trois ans après en "finance and business management ", qualification reconnue nationalement ${ }^{27}$, elle continue à travailler dans l'équipe de ventes. Mais en 1995, son bureau de Norwich est fermé suite à une réorganisation, et elle en profite pour quitter l'entreprise en acceptant un licenciement "volontaire » car c'est l'occasion de faire le tour du monde avec son compagnon de l'époque (elle se séparera pendant le voyage). Quand elle revient un an après, en 1997, elle retrouve très vite via une mission d'intérim un emploi à Yorkshire Electricity, comme commercial pour les clients industriels. Un an après, elle intègre le prestigieux et innovant département commercial ( special markets»), où ils proposent de négocier des contrats «à façon» («bespoke contrats») pour des industriels qui participent à gérer la charge sur le réseau.

Quand l'entreprise est rachetée par Npower en 2001, elle est pour la deuxième fois licenciée sur motif personnel car elle refuse de déménager à Swindon. Alors qu'elle a une offre d'emploi de British Petroleum, elle préfère accepter la proposition de son ancien chef d'intégrer une petite structure de commercialisation où elle arrive avec ses clients industriels qui la suivent. S'ensuit quatre ans d'intense activité pour développer la structure, avec des horaires assez fous (7h30-19h sans pause), où elle devient à 30 ans responsable régional des ventes, responsable de sept collaborateurs, "une progression naturelle pour moi » et où elle tombe aussi amoureuse de son ancien (et actuel) chef.

A son retour de congé de maternité, elle réussit à conserver son poste de manager tout en négociant un temps partiel de 3 jours par semaine. Mais sa charge de travail n'a pas été allégée et elle revient rapidement à 4 jours par semaine, tout en avouant souvent travailler de chez elle au téléphone ou par mail le vendredi. Lors de la dernière réorganisation, en 2006, elle vient d'être rétrogradée de manager à simple commerciale, le management ayant anticipé son prochain départ en congé de maternité (elle ne sera pas remplacée). Elle reconnaît qu'elle ne pouvait pas assumer les attentes de la direction, qui souhaite des cadres très investis ( strongly committed ») avec des horaires extensifs à l'image de ceux de son mari (7h-19h), car cela empiète leur vie privée. Habitant à la campagne, leur fils de 2 ans est par exemple à la crèche à côté de l'entreprise, il fait donc plus d'une heure et demi de transport avec eux et reste parfois jouer le soir dans les couloirs en attendant la fin d'une réunion. Enceinte du deuxième, elle reconsidère lors de l'entretien en 2006 ses ambitions de carrière, même si elle souhaite continuer à travailler à temps partiel, notamment pour le train de vie confortable que deux salaires de managers procurent. Deux ans après, Anna a démissionné pour suivre son mari recruté dans une nouvelle division au siège à Paris pour une mission de trois ans. Elle s'occupe de ses enfants à temps plein.

\footnotetext{
${ }^{25}$ Cet écart qui s'est pratiquement résorbé dans les jeunes générations, avec $46 \%$ en Hongrie contre 44\% en Angleterre de jeunes femmes parmi les «managers and professionals » de moins de 35 ans.

${ }^{26}$ Via un " training program », système très développé en Angleterre, où les jeunes sont salariés pour trois ans et souvent « tournent» sur des postes tous les 4 ou 6 mois. Ils bénéficient d'heures libérées pour préparer leurs examens et parfois pour suivre leurs cours.

${ }^{27}$ Par la CIMA, Chartered Institute of Management Accountants.
} 
Malgré cette féminisation des premiers niveaux de responsabilité, et donc davantage de candidates pour la promotion, les niveaux supérieurs de direction leur sont encore relativement fermés dans les deux pays. Depuis le rachat, le nombre de femmes dans l'équipe dirigeante est en effet resté similaire en Hongrie ( 2 femmes parmi les 13 directeurs) et a même diminué en Angleterre (les 3 dirigeantes de l'entreprise de Telford, dont la PDG, ont été licenciées, et ont été remplacées par des hommes). Ce maintien du plafond de verre dans des organisations profondément remaniées échappe d'ailleurs en partie aux acteurs en place car les femmes sont en nombre croissant parmi les jeunes cadres. Il leur échappe aussi car les systèmes informatiques de gestion du personnel sont eux aussi déstabilisés par ces rachats et réorganisations, et ne permettent pas de suivre les mobilités intra et inter-organisationnelles dans la durée. Aucune des deux filiales n'avait ainsi sexué les statistiques de turn-over, qui s'avère pourtant plus élevé pour les femmes dans les deux filiales. Loin d'avoir modifié les règles du jeu pour faire carrière, cette contraction du marché interne n'a-t-elle pas en fait renforcé un modèle "masculin-neutre » d'activité, conduisant à une progressive éviction des femmes de la course au pouvoir, à l'image d'Anna ?

\section{Le renforcement d'un modèle «masculin-neutre» d'activité dans des marchés internes du travail en contraction}

$\mathrm{Si}$ les effets de recomposition des espaces de carrière semblent offrir des opportunités nouvelles mais très sélectives pour les femmes, le processus de libéralisation du secteur énergétique a aussi eu des effets de dérégulation du marché du travail que l'on peut saisir à l'aune du renforcement de certaines « normes » masculines de carrière.

\subsection{La mobilité géographique : entre contraintes de relocalisation et perspectives sélectives d'expatriation}

Au niveau local, les salariés doivent souvent faire face à des contraintes de mobilité accrue avec des transferts (individuels ou d'équipe). La forte dispersion de l'implantation géographique des entreprises du secteur de l'énergie sur les territoires nationaux se conjugue en effet avec les effets spécifiques des restructurations entraînant fréquemment la délocalisation/relocalisation de certaines catégories de salariés, cadres ou non cadres. Dans les deux filiales étudiées, les fonctions support et dirigeante ont été particulièrement touchées par les contraintes de mobilité géographique ${ }^{28}$. En Hongrie, suite à la fusion des deux entreprises, les responsables des services RH et Finance ont dû choisir de localiser leur équipe à Györ ou à Szeged (à plus de $200 \mathrm{~km}$ au Nord ou au Sud de Budapest). De même en Angleterre, le choix du groupe d'implanter la filiale à Leeds a contraint les salariés de l'entreprise localisée à Telford à accepter de travailler à Leeds ou à négocier leur départ : sur 47 salariés licenciés cette annéelà, 31 étaient des femmes (soit 2/3). Les femmes semblent plus particulièrement touchées par les contraintes de mobilité induites par les restructurations (Collins, 2005). Souvent employées dans des fonctions support, elles sont particulièrement concernées par les effets de centralisation de ces fonctions vers le siège et la

\footnotetext{
${ }^{28}$ Car les postes techniques des salariés d'exécution ont souvent été supprimés ou externalisés sur place... Dans le cas d'une restructuration d'un groupe pharmaceutique, le transfert (interne) vers d'autres sites a concerné les emplois qualifiés de R\&D, alors que l'externalisation (accompagnée d'un déclassement statutaire et salarial) a touché les emplois peu qualifiés : courrier, standard, propreté (Guyancourt, 2007).
} 
difficulté de maintenir leur activité en local. L'un des objectifs des restructurations étant de rationaliser les structures organisationnelles, de se recentrer sur le « cœur de métier» et de réduire les coûts liés aux activités «non productives », la chasse aux doublons expose les fonctionnels à un risque accru de licenciement ou de relocalisation (Karambayya, 1998). En Hongrie, l'injonction à la mobilité est vécue comme plus contraignante qu'en Angleterre où le marché de l'emploi est globalement dynamique. Etre mobile au niveau local devient une garantie, certes toujours provisoire, de garder son poste.

Dans tous les pays, les cadres optent souvent pour une stratégie de double résidence (professionnelle et familiale) et le célibat géographique en semaine. En filiales, la mobilité géographique sous contrainte se heurte en effet à la force de l'enracinement local, tant du point de vue des attaches familiales et culturelles que des contraintes économiques liées au coût de l'immobilier, variable selon les régions, à la faiblesse du marché locatif et à l'emploi du conjoint. En Hongrie, le modèle du couple à double salaire (et l'emploi fréquent des épouses dans le secteur public), le recours à l'entraide familiale pour la garde des enfants et la disparité du coût de la vie selon les régions constituent des entraves importantes à la mobilité des cadres - hommes ou femmes, comme le rappelle le cas de Beata plus loin. En Angleterre, les difficultés d'accession à la propriété et l'attachement à la région expliquent principalement le refus de la mobilité, y compris pour les célibataires. La qualité de vie dans le Yorkshire est souvent mise en avant pour justifier l'ancrage local, en dépit de configurations familiales plutôt favorables à la mobilité. La plupart des cadres dirigeants ont en effet des conjointes qui ont arrêté de travailler (ou s'apprêtent à le faire) pendant de nombreuses années pour élever leurs enfants ou optent pour un travail à temps partiel. Comme le soulignent d'autres travaux, le rôle de la configuration familiale (ne pas avoir d'enfant), de la qualification (élevée) et du logement (être locataire) sont centraux dans l'acception de la mobilité géographique (Vignal, 2005). La variable du genre n'est pas sans influence non plus (Bertaux-Wiame, 2006 ; Guillaume, Pochic, 2008).

\section{Beata ou comment garder à tout prix son équilibre familial}

Beata est diplômée de l'Université de Szeged, une des cinq grandes villes de la Hongrie, à côté de la frontière serbe et non loin de la frontière roumaine. De 1985 à 1990, elle a suivi 5 ans de formation juridique et 2 ans de formation économique. Beata parle également trois langues étrangères (français, allemand, anglais). Elle a appris le français dans la seule école de Szeged où cela était possible car sa mère professeur de philosophie tenait à ce qu'elle ait une culture générale. Elle souhaitait être avocate, mais n'a pas pu obtenir de poste à cause du numerus clausus. Elle a ensuite commencé à travailler à l'université de Szeged comme enseignante en droit constitutionnel et en même temps au tribunal pour préparer son examen d'entrée dans la magistrature, mais malgré sa première place, elle est découragée à le faire car le tribunal a d'autres candidats "protégés" à placer (proximités politiques et clientélisme local). Elle décide finalement de partir travailler dans le secteur privé, d'autant que le salaire qu'on lui propose est nettement supérieur. Le dirigeant d'une entreprise de chimie qu'elle avait connu au travers de travaux de traduction qu'elle avait réalisés pour lui, lui propose en effet de devenir DG d'une petite structure ( 80 personnes) à $30 \mathrm{~km}$ au nord de Szeged. Elle y reste 5 ans, se marie et a son premier enfant.

En 1997, quand elle voit une annonce dans le journal pour un poste de DRH chez EGAZ, elle postule parce qu'elle parle le français, que le poste l'intéresse et qu'il se trouve à Szeged. Son mari fait lui-même une carrière de DRH dans le secteur privé dans des entreprises multinationales. En 1998, elle a son deuxième enfant. En 2000, suite à la fusion d'EGAZ et DEGAZ, elle devient DRH pour les deux entités. Son bureau est situé à Szeged au Sud du pays, mais elle partage son temps entre les trois sites de l'entreprise, Szeged, 


\begin{abstract}
Gÿor dans le Nord et Budapest (siège social de l'entreprise), chaque site étant distant de 200 $\mathrm{km}$. Depuis qu'elle a été nommée, l'entreprise n'a cessé de se transformer, d'abord la fusion entre les deux entreprises régionales, puis les réductions d'effectifs, les externalisations... Son mari a changé plusieurs fois d'entreprises et travaille aujourd'hui à Budapest. Il vient à Budapest le lundi matin et il revient le jeudi soir ("si on a de la chance») ou le vendredi soir ("si on n'a pas de chance»). Quand ils sont absents tous les deux, c'est la famille élargie qui s'occupe des enfants (grands-parents, sœur, oncle, tante). Tous habitent dans la même rue à Szeged, «les enfants sont à la maison chez mes parents, chez ma sœur aussi ». Interrogée sur l'idée d'arrêter de travailler, elle rétorque qu'il s'agit d'une question d'Europe de l'Ouest. En Hongrie, les couples ont besoin d'un double salaire et il est impossible pour une femme de recommencer une carrière à 45 ans. Et puis elle aime bien son travail, malgré les diminutions d'effectifs. Pour le moment, elle n'envisage aucune mobilité professionnelle, le contenu de son travail ne cesse d'évoluer et la carrière de son mari ne lui permet pas d'aller travailler ailleurs dans le Groupe, sauf s'ils avaient une proposition commune. Sa priorité, c'est son équilibre familial. Deux ans après, elle est toujours au même poste.
\end{abstract}

Si certains hommes cadres anglais ou hongrois se vont vus offrir des opportunités de mobilité internationale, rares sont ceux qui sont allés à l'étranger avec leur famille. Les rares exemples d'impatriation dans la maison-mère se sont révélés être des échecs : missions de courte durée, sans perspective de promotion sur place, avec des conditions financières peu attractives, des difficultés à manager une équipe ou à faire valoir son expertise dans une langue étrangère et enfin une intégration sociale limitée du côté des collègues. Pourtant, paradoxalement, construire une partie de sa carrière à l'international devient un critère de distinction dans un contexte où les espaces de carrière sont en pleine recomposition. Plus que les atouts linguistiques, l'acceptation d'une hypothétique mobilité au sein du groupe devient un critère officiel de détection de cadres «à potentiel » en filiales, inscrit dans les grilles d'évaluation. Les (rares) opportunités d'expatriation-impatriation sont donc l'objet de stratégies individuelles réservées in fine aux hommes cadres dirigeants qui peuvent négocier âprement les termes de leur contrat, en échange d'une disponibilité familiale (la plupart ont une femme inactive ou sont célibataires) et d'un investissement professionnel intense.

\title{
2.2. Des filiales sous pression, des exigences de disponibilité renforcées
}

Au-delà des questions de délocalisation, les contextes de fusion-restructuration sont propices à la dérégulation des horaires de travail (Crompton, 2006 ; Wajcman, 1998) notamment en raison de la faible prise en compte de l'activité de travail concrète (Raveyre, 2005) et des fréquentes réductions d'effectif. Qu'il s'agisse du démarrage d'une activité comme dans le cas de la filiale anglaise ou d'une recherche accrue de productivité dans le cas hongrois, l'extension des horaires de travail se fait particulièrement sentir sur les salariés, et particulièrement les cadres et les femmes ${ }^{29}$. La diffusion d'une pression aux résultats et d'une logique fortement entrepreneuriale, dans un contexte de stricte supervision par la maison-mère, offre des contextes de travail vécus comme stimulants - en particulier pour les jeunes diplômés qui sont «à bonne école » et sont à la recherche d'une première expérience managériale - et usants quand la pression se maintient dans la durée.

\footnotetext{
${ }^{29}$ «Il semble évident que se fonder sur les heures de travail comme signe de l'investissement au travail peut entraîner aussi bien une « foire d'empoigne » (c'est-à-dire trop d'heures pour tous) et un biais de sélection envers les femmes » (Osterman, 1996, p. 16, traduction des auteurs).
} 
Si en Angleterre, le contexte économique favorable offre aux salariés la possibilité de poser des limites à un investissement professionnel jugé excessif ${ }^{30}$, la situation des cadres hongrois semble moins propice à la modération des horaires de travail. Confrontés à la réduction constante des effectifs et à l'accroissement de leur charge de travail lié à l'élargissement de leur périmètre de responsabilité, les cadres dirigeants ironisent parfois sur le décalage entre la tranquillité de la maison-mère et la surcharge chronique et persistante des salariés de la filiale. Plus généralement, la situation économique de la Hongrie depuis le changement de régime a conduit les cadres qui voulaient se maintenir dans leur emploi ou en trouver un, à privilégier un investissement professionnel intense. Nombre d'entre eux maîtrisent plusieurs langues, ont repris ou fait des études très poussées (double diplôme) et tentent de mettre tous les atouts de leur côté pour garder une «place au soleil». Les contextes de rachat/restructuration ne font qu'intensifier un engagement déjà prioritaire dans la sphère professionnelle, estompant les frontières entre travail et hors travail et déstabilisant les régulations collectives existantes (Linhart, 2005).

Dans ce contexte, les femmes cadres souvent en couple à double carrière et $a$ fortiori les mères de famille - moins mobiles et moins disponibles - sont rapidement confrontées à un choix entre leur carrière ou leur famille, alors que les cadres masculins sont généralement caractérisés par un soutien domestique à leur carrière (Wajcman, 1996). Dans les deux filiales, cette équation est influencée par le « régime de genre » (Lewis, 1997) qui structure la division sexuelle du travail. Bien que les deux pays soient classés différemment par les typologies existantes (Crompton, 1997; Lewis, 1992; Esping-Andersen, 1990), l'Angleterre correspondant à un modèle «libéral » et la Hongrie à un modèle «postsocialiste $»^{31}$, le rôle prépondérant des femmes dans la prise en charge du travail domestique et l'éducation des enfants permet de comprendre leurs difficultés à se conformer aux normes de carrière. En Angleterre, la faiblesse des modes de garde collectif avant l'âge de 6 ans et surtout leur coût contribuent à dévaloriser l'intérêt financier d'une activité professionnelle à temps plein pour les mères de famille. Si les femmes cadres tentent de se maintenir dans l'emploi avec un premier enfant au prix de gros sacrifices financiers, elles optent souvent pour un temps partiel ou un congé parental de longue durée au moment de leur seconde grossesse. Le recours au temps partiel étant perçu comme incompatible avec une activité managériale, certaines acceptent même d'être rétrogradées ou se maintiennent volontairement dans des activités d'expertise, au prix d'un plafonnement de carrière. En Hongrie, l'effritement, à partir de 1985, du modèle «maternaliste » (Haney, 2002) qui visait en théorie à couvrir les besoins des femmes en tant que mère et travailleuse à temps plein est en partie compensé par l'existence de modes de garde collectifs pour les enfants de plus de 3 ans $^{32}$ (Fodor et al, 2002), mais se heurte à la permanence d'une vision traditionnelle des rôles sociaux des hommes et des femmes (Nagy, 2005). Les femmes cadres interviewées, le plus souvent en couple

\footnotetext{
${ }^{30}$ au grand dam du directeur français de la filiale qui travaille fréquemment jusqu'à 22 heures et s'offusque des horaires de travail « insuffisants », $8 \mathrm{~h}-18 \mathrm{~h} 30$, des membres anglais de son équipe de direction...

${ }^{31}$ Le temps partiel étant presque inexistant en Hongrie (alors qu'il est la forme majoritaire de l'emploi des mères de famille), et les femmes peu qualifiées étant fortement exclues du marché du travail (alors qu'elles y restent davantage en Angleterre), cf. tableau 2.

32 et d'autres services privés ou avantages fiscaux à destination des familles de classes moyennes.
} 
à double carrière, tentent donc de « concilier 》 travail et famille avec le soutien de leur entourage familial, mais les plus jeunes envisagent fréquemment le recours au congé parental long (GYES, jusqu'à 3 ans) hérité du régime communiste. Le temps partiel, considéré comme un demi-salaire (alors que les salaires sont considérés comme faibles par rapport au coût de la vie), est pratiquement inexistant.

Si des opportunités de carrière se sont ouvertes pour les femmes, notamment dans des sphères d'expertise nouvelles, le renforcement des normes de carrière au masculin lié au contexte de restructuration contribue indéniablement à consolider le plafond de verre dans les deux filiales étudiées. Pourtant, en Angleterre, avant la fusion-acquisition, trois femmes étaient cadres dirigeantes, dont l'une directrice d'une des deux entreprises rachetées. Peut-on imputer aux seules contraintes de mobilité (déménagement imposé vers Leeds) la seule responsabilité de leur éviction?

\section{La reconduction des mécanismes de cooptation : l'entre soi masculin}

Sans doute faut-il également rechercher une interprétation du côté des règles informelles de gestion des carrières, et de la reconduction à une autre échelle de mécanismes de cooptation.

\subsection{Se rendre « visible » dans un contexte d'incertitude pour tous}

De nombreux travaux ont souligné l'importance de l'évaluation par les pairs et de la cooptation dans le déroulement de la carrière en entreprise, permettant d'expliquer en dynamique la (re)fabrication de l'homogénéité sociale et sexuée des dirigeants (Guillaume, Pochic, 2007; Fortino, 2002; Wajcman, 1998; Kanter, 1977). Or l'un des effets évidents des restructurations est précisément de déstructurer les réseaux relationnels internes et de casser les relations de confiance (ou au moins d'interconnaissance) qui s'étaient tissées au fil du temps entre les salariés et leur hiérarchie, créant des situations de vulnérabilité (Rorive, 2005) et « d'incertitude pour tous 》 (Linhart, 2002). La recomposition quasi-systématique des équipes dirigeantes (départ à la retraite anticipée de l'ancienne direction de la filiale hongroise et licenciements de la moitié des dirigeants dans la filiale anglaise), avec l'arrivée de nouveaux responsables issus de l'entreprise qui rachète contribuent à remettre en question les compromis relationnels construits entre salariés et hiérarchie et à rendre plus imprévisible les normes d'évaluation du travail. Avoir des contacts privilégiés avec des responsables hiérarchiques (anciens collègues ou anciens supérieurs) facilite l'accès à des informations et des soutiens pour éviter certains pièges difficiles à anticiper, en raison d'une modification continuelle de la hiérarchie symbolique (et pratique) des services et des postes, de la stratégie et du périmètre des organisations ${ }^{33}$.

\footnotetext{
${ }^{33}$ Pièges comme par exemple rester dans un service qui va être externalisé ou dont les échelons hiérarchiques sont réduits, devenir un expert dans un domaine en perte de vitesse ou sur lequel existe un doublon. Ou à l'inverse se faire financer un deuxième diplôme sur une spécialité en vue, ou intégrer une petite structure qui se développe et crée des échelons supplémentaires, etc...
} 
Dans les deux filiales, les cadres qui se sentent les plus confiants à l'égard de leur futur déroulement de carrière sont donc ceux qui savent détenir une compétence stratégique évidente pour la filiale (métier cible ou grande connaissance du secteur au plan national) ou qui parviennent à nouer des relations avec le Groupe, notamment par un lien privilégié avec un expatrié ou un cadre français de la maison-mère, construit à travers des relations de travail. Ceci exclut un certain nombre de cadres qui ne parlent ni le français ni l'anglais et dont l'activité est peu visible ou peu mesurable pour le Groupe comme par exemple cette femme chargée des questions de réglementation et (ré)élue dans les instances professionnelles du secteur énergétique anglais.

\section{Linda, une brillante autodidacte de la régulation du marché}

A 49 ans, Linda est responsable des relations avec le régulateur (« regulatory affairs manager ») de la filiale anglaise et gagne environ $50.000 £$ par an. Issue d'un milieu modeste, orpheline jeune élevée par sa grand-mère, elle a commencé à travailler à 17 ans comme employée administrative dans des services municipaux chargés du logement social. Mais après la naissance de sa deuxième fille, elle décide à 32 ans de reprendre des études supérieures d'informatique pendant trois ans et est embauchée à Northern Electric après son stage d'études en 1988, au moment du tournant commercial de cette entreprise publique régionale dominée par des ingénieurs. Si elle commence comme administrateur de bases de données, elle évolue assez rapidement vers le monde de la régulation qui se met en place avec la libéralisation du secteur de l'énergie, où elle est chargée de connaître les dernières lois et réglementations, les acteurs du régulateur $\left(\mathrm{OFGEN}^{34}\right)$, et de faire du lobbying auprès des professionnels du secteur et du monde politique. Elle représente ainsi l'entreprise dans divers comités et associations, à Londres et dans tout le pays. Elle y rencontre des cadres de Yorkshire Electricity, qui la débauche en 1997 pour s'occuper de leur représentation externe ( representation manager »), et elle accepte car c'est à la fois une reconnaissance par ses pairs de sa valeur et surtout car ses cadres sont beaucoup plus innovants et commerciaux que les vieux ingénieurs de l'ancienne entreprise publique. Son plus gros client part d'ailleurs avec elle. Même si l'entreprise est basée à Leeds, elle ne déménage pas du North East ; avec ses déplacements, elle ne passe que deux jours en moyenne au bureau, et ne veut pas déraciner ses deux enfants. Son mari travailleur social, aidé de sa propre mère, s'en occupe pendant ses absences. Elle grimpe rapidement les échelons chez Yorkshire Electricity, dirige un service de onze personnes et se retrouve membre du comité exécutif, élue l'employée de l'année. Elle obtient également une reconnaissance nationale en étant élue première femme représentante de l'industrie dans les instances de régulations nationales (Elexon et BSC Panel ${ }^{35}$ ). Son couple par contre souffre de cette progressive différence de réussite sociale : ils se séparent, « et je pense que sa mère y est pour quelque chose, cela devait jaser derrière mon dos : Et Barbara qui n'est jamais là... »

Quand en 2000, quand Npower rachète son entreprise, elle ne veut pas travailler pour eux à la fois parce qu'elle ne partage pas les mêmes valeurs que ses dirigeants et aussi parce qu'elle pense qu'elle ne pourrait pas conserver la même position de pouvoir. Elle est donc licenciée « volontaire » à 45 ans et reste trois mois au chômage pour la première fois de sa vie, ce qui la terrifie. Heureusement son ancien directeur lui propose de rejoindre une nouvelle entreprise que vient de créer un petit groupe d'anciens de Yorkshire Electricity, "ils savaient qu'ils obtiendraient un haut degré d'exposition dans le secteur." Elle accepte alors une diminution de salaire et de statut, puisqu'elle n'a qu'un assistant alors que les grandes entreprises ont des départements entiers chargés des relations avec le régulateur. $\mathrm{Au}$ sein de cette filiale de commercialisation, rachetée deux ans plus tard par l'entreprise française étudiée, elle pense pourtant qu'elle n'a aucune opportunité de promotion : «Je suis femme, ce qui est un gros handicap. Je ne suis pas française, je ne suis pas basée à

\footnotetext{
${ }^{34}$ Office of Gas and Electricity Market

${ }^{35}$ Le BSC, Balancing and Settlement Code, contient les règles et principes de gouvernance que doivent signer les entreprises d'électricité licenciées, et est mis en œuvre sous le contrôle d'une entreprise indépendante (Elexon) et d'un panel d'industriels (BSC Panel).
} 
Paris et je ne suis pas visible ». Deux ans après l'entretien, elle démissionne pour aller travailler dans une instance de régulation où elle siégeait.

Davantage présentes dans les premiers niveaux de responsabilité ou dans des métiers de back-office, malgré parfois leurs compétences linguistiques, les femmes cadres ont moins l'occasion de se construire un réseau relationnel efficace, de se rendre visibles des «faiseurs de carrière » (Guillaume, Pochic, 2007).

\subsection{La recomposition des réseaux, à une autre échelle}

Au-delà des relations avec le groupe actionnaire, les contextes de restructuration recomposent les réseaux relationnels, mais sur une maille à la fois plus resserrée (l'équipe de dirigeants) et plus étendue (les acteurs du marché professionnel). Ils rendent aussi plus visibles la nécessité de ressources relationnelles, désormais inter-organisationnelles, pour continuer à progresser à travers des mobilités externes, avec ou sans épisode de chômage (Martin, 2005 ; Woodall et al., 1997). Le cas anglais est très illustratif de cette recomposition. Une partie des salariés de la filiale dont trois dirigeants avaient travaillé ensemble au sein de Yorkshire Electricity, dans un service commercial reconnu comme excellent, mais pourtant supprimé, avant de participer ensemble au lancement de la "spin off» qui sera ensuite rachetée par l'entreprise française. Ces cadres dirigeants ont attiré dans leur sillage et parfois débauché un certain nombre des jeunes cadres qu'ils encadraient à l'époque de Yorkshire Electricity. Cette expérience professionnelle commune s'est ensuite prolongée au fil des rachats successifs et contribue aujourd'hui à la formation d'une « solidarité de clique » (Dalton, 1959) au sein de la filiale. Parallèlement, la libéralisation du marché énergétique et la disparition des grandes entreprises a contribué à la dispersion des cadres les plus attachés au secteur au sein de différentes structures de taille moyenne et à l'explosion d'un marché du conseil spécialisé dans l'énergie. Les réseaux semblent moins fondés sur la base du diplôme et des instituts de formation fréquentés (en formation initiale ou continue) que sur la base d'une expérience professionnelle commune (au sein d'entreprises ou d'associations professionnelles), capital expérience qui met en porte-à-faux les plus jeunes, et notamment les femmes.

En Hongrie où le marché de l'énergie est encore fortement régulé par l'Etat, mais dans un contexte de privatisation massif depuis 1989, les ressources relationnelles sont d'une autre nature. Nous avons déjà évoqué le poids du capital politique avant la transition, le Parti communiste ayant son mot à dire sur toutes les promotions dans les entreprises d'Etat dirigée par un "carré de décision » (la direction, le Parti, le syndicat unique et le mouvement de la jeunesse communiste $^{36}$ ), poids en recul relatif depuis les années 1980. La carrière des cadres dirigeants issus de l'ancienne entreprise d'Etat reste cependant étroitement liée à leur réseau politique, tant au niveau des instances nationales de régulation du marché que du Ministère. Comme en France, la spécificité du marché professionnel de l'énergie contribue par ailleurs à la formation d'un cercle restreint de cadres qui ont souvent été formés dans les mêmes universités en

\footnotetext{
${ }^{36}$ Le KISZ, Kommunista Ifjúsági Szövetség, quasi-obligatoire, était un lieu de détection des talents d'organisation, futurs cadres du Parti ou du syndicat ( $k$ kader »), mais aussi cadres des entreprises ou de l'administration. De nombreux leaders politiques ou syndicaux en poste sont issus de cette filière du KISZ, comme l'actuel Premier Ministre Gyurcsány Ferenc.
} 
formation initiale ou continue ${ }^{37}$ et peuvent s'appuyer sur des réseaux d'anciens élèves. Du fait de la prépondérance des formations d'ingénieurs dans la hiérarchie professionnelle, et de la faible attractivité du secteur gazier pour les jeunes femmes qui choisissent des diplômes techniques ${ }^{38}$, ces phénomènes de cooptation jouent en faveur des hommes (et des très rares femmes) qui ont des qualifications ou compétences étroitement liées au secteur d'activité. Si les universités de droit ou de commerce qu'elles ont fréquentées, plus récentes, produisent elles aussi des anciens élèves, ces réseaux n'ont pas la même histoire ni la même efficacité que les universités techniques traditionnellement liées au secteur énergétique (université de Miskolc pour le gaz et université de Veszprém pour l'électricité et le pétrole $\left.{ }^{39}\right)$, comme le révèle la carrière de Csaba.

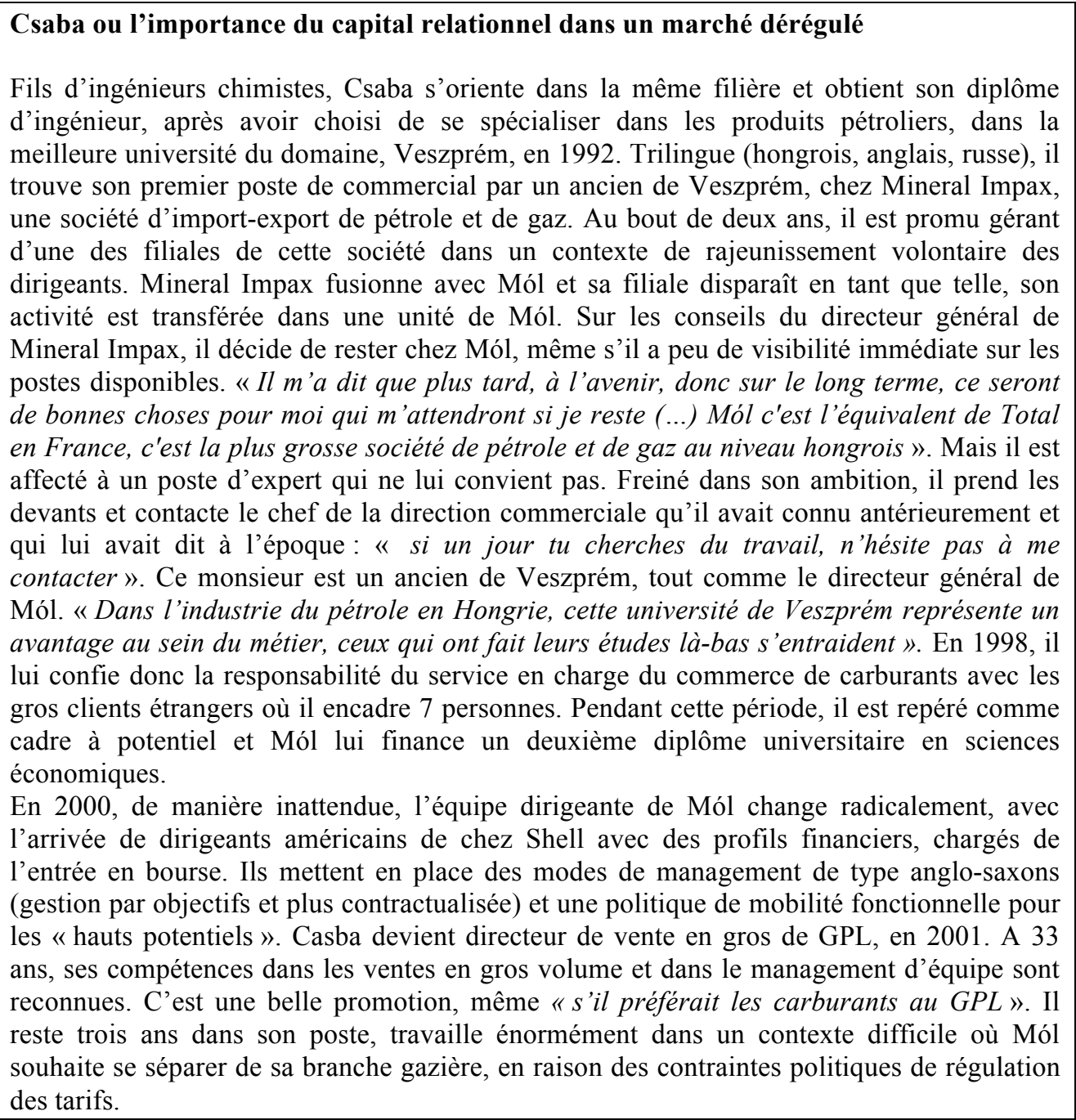

\footnotetext{
${ }^{37}$ L'université de Miskolc en Hongrie est fortement soutenue par les grandes entreprises gazières qui lui versent une « taxe d'apprentissage » et envoient régulièrement des stagiaires.

${ }^{38}$ A peine une femme par promotion à Miskolc. Comme le montrent d'autres travaux, le nombre de femmes ingénieurs n'a cessé de croître dans ces deux pays, mais les jeunes femmes ne s'orientent pas dans ce secteur d'activité, privilégiant d'autres spécialités, comme la biologie ou la géologie.

${ }^{39}$ Créées en 1949, ces deux universités techniques situées respectivement au Nord-Est et au NordOuest de la Hongrie ont été des piliers de la politique d'industrialisation du pays, via leurs spécialités d'ingénierie dans l'industrie lourde (pétro-chimie, métallurgie, nucléaire, puis informatique) et comptent parmi les plus grandes universités en terme de nombre d'étudiants.
} 
A l'occasion d'un dîner d'affaires avec le responsable commercial de la filiale du groupe
français étudié, un expatrié français qu'il connaît bien, il évoque son souhait de quitter Mól
tout en capitalisant sur son expertise gazière. Il est fortement recommandé au Directeur
Général Adjoint français de la filiale, qui a le projet de racheter cette branche. On lui
propose de lancer une petite filiale en charge du marché libéralisé. Suite au départ soudain
du directeur commercial, il bascule sur son poste, après avoir passé une évaluation
satisfaisante à Paris. En 2006, il envisage de partir travailler dans une autre filiale
anglophone du groupe et a des contacts personnels avec la filiale anglaise. Il vient juste
d'être père à 37 ans de jumeaux. Sa femme experte fiscale est en congé parental et
n'envisage pas de retravailler avant quelques années. Il considère d'ailleurs qu'ils ont un
peu sacrifié leur vie familiale pour leurs carrières, " quand le capitalisme farouche est
arrivé en Hongrie, les jeunes qui ont démarré dans les années 1990 ont eu des opportunités
formidables, mais... la famille a été un peu reléguée au deuxième plan ». Deux ans après
notre passage, il a d'ailleurs été promu et est désormais n³ de la filiale, sa femme est
encore en congé parental long.

D'une certaine façon, par la cooptation, les cadres tentent de pallier les effets de la dérégulation du marché du travail et la déstabilisation des modes de socialisation professionnelle partagés, se protégeant en pratique de la réalité d'un marché du travail libéral (Courpasson, Dany, 1994). Dans un contexte de restriction des espaces d'opportunité et de luttes des places, on peut comprendre que les rares femmes qui avaient réussi à accéder aux fonctions dirigeantes dans les grosses entreprises du secteur aient eu du mal à s'y maintenir (nombre d'études montrent que la féminisation des strates supérieures est plus facile dans les contextes de croissance des effectifs que l'inverse). L'éviction de toutes les femmes membres de l'équipe de direction, y compris la DG, au moment de la fusion des deux entreprises en Angleterre est de ce point de vue assez éclairant. Rarement évoqué ce fait est justifié par l'un des dirigeants actuels par une expression on ne peut plus signifiante : "we were confortable with each other » (les dirigeants hommes issus de la spin off). Confort que l'on peut interpréter comme une recherche d'efficacité fonctionnelle (routines de travail partagées), le partage de valeurs communes (la culture Yorkshire Electricity), mais confort que l'on peut difficilement extraire du contexte d'homophilie (Kanter, 1977) qui le caractérise.

Du fait de la ségrégation scolaire et professionnelle, les femmes n'occupent que très rarement des emplois "spécifiques » à l'industrie du gaz. Elles ont à la fois plus d'opportunités de transférer leurs compétences à d'autres secteurs (dans les deux pays le secteur énergétique n'est d'ailleurs pas considéré comme un secteur particulièrement attractif pour les jeunes diplômés d'écoles de commerce ou universitaires non techniques) et moins de chances d'être repérées pour certains postes du fait de leur moindre visibilité dans les lieux de détection propres au secteur (écoles spécialisées, associations professionnelles, instances de régulation) et du poids relativement faible (tant sur le plan numérique que symbolique) de leurs métiers au sein des entreprises. Ce qui explique sans doute leur plus forte probabilité de sortir en cours de route de la course à la carrière ${ }^{40} \ldots$

\section{Conclusion}

Cette recherche nous invite à conclure que les effets sur les carrières des femmes cadres des processus de fusion-acquisition étudiés sont en réalité plutôt négatifs. La recomposition des espaces d'opportunité liée à l'internationalisation du groupe

\footnotetext{
${ }^{40}$ D'après nos calculs, les femmes représentent $47 \%$ des démissionnaires sur 5 ans alors qu'elles ne sont que $33 \%$ des salariés de la filiale.
} 
et la dérégulation du marché contribue certainement au développement de filières de carrière plus ouvertes aux cadres non détenteurs des atouts de départ anciennement indispensables (diplôme d'ingénieur dans les trois pays et capital politique/origine sociale modeste en Hongrie). Cette ouverture offre la possibilité aux femmes de combler en partie leur retard structurel lié à leur faible présence dans les écoles d'ingénieur, mais aussi à leur moindre participation aux réseaux et instances du Parti en Hongrie (Fodor, 2002). Cette féminisation se caractérise néanmoins par une forte ségrégation professionnelle et un plafonnement de carrière. Le renforcement d'un modèle de carrière au masculin, caractérisé par des exigences de mobilité contrainte notamment pour les fonctions support très féminisées et de disponibilité extensive pour tous les métiers, contribue parallèlement à durcir les normes formelles de carrière déjà identifiées comme particulièrement discriminantes pour les mères de famille (Wajcman, 1996; Wajcman, 1998 ; Laufer, 2004 ; Laufer, 2005). Nombre de jeunes femmes cadres étiquetées à potentiel sont donc aujourd'hui au seuil du plafond de verre dans les deux filiales. Une des questions qui se pose aujourd'hui à tous les salariés hommes ou femmes - est de savoir s'ils vont accepter de (et pouvoir) se conformer à des modèles de carrière de plus en plus exigeants, dans un contexte où se combine forte incertitudes sur l'avenir et nécessité de s'appuyer sur des ressources relationnelles solides.

\section{Bibliographie}

Beaujolin, R., 1999. Les vertiges de l'emploi. L'entreprise face aux réductions d'effectifs. Grasset/Le Monde, Paris.

Bender, A.F., Cadin, L., de Saint-Giniez V., 2003. Carrières nomades, les enseignements d'une comparaison internationale. Vuibert, Paris.

Bertaux, D., 1996. Les récits de vie. Nathan 128.

Bertaux-Wiame, I., 2006. Conjugalité et mobilité professionnelle : le dilemme de l'égalité. Cahiers du Genre (41), 49-73.

Boltanski, L., Chiapello, E., 1999, Le nouvel esprit du capitalisme. NRF Gallimard, Paris.

Bunel, M., Duhautois, R., Gonzales, L. 2007. L'emploi lors des fusionsacquisitions de la fin des années 1990, Connaissances de l'emploi, 48, novembre.

Clot, Y., 1994. Licenciements et travail psychique: l'exclusion à l'intérieur. Chimères printemps, 71-90.

Collins, G., 2005. The gendered nature of mergers. Gender, Work and Organization, 12 (3), 270-290.

Courpasson, D., Dany, F., 1994. La gestion des carrières des cadres en question. Gérer et comprendre. Annales des mines, septembre, 30-44.

Crompton, R., 1997. Women and work in modern Britain, Oxford University Press.

Dalton, M., 1959. Men who manage. Fusions of feeling and theory in administration. John Wiley \& Sons, New York. 
Paru dans le dossier « Le plafond de verre dans les professions supérieures »

(coord. Catherine Marry, Marie Buscatto), Sociologie du travail, vol.51, n², 2009, p. 275-299.

Dejours C., 1998. Souffrance en France. La banalisation de l'injustice sociale, Le Seuil, Paris.

Devine, F., Britton, J., Mellor, R., Halfpenny, P., 2000. Professional work and professional careers in Manchester's business and financial sector. Work, employment and society. 14, 3, 521-540.

Doeringer, P., Piore, M., 1971. Internal labor markets and manpower analysis. D.C. Heath, Lexington.

Esping-Andersen, G., 1990. The three worlds of welfare capitalism, Cambridge, Polity.

Fodor, E., 2003. Working differences: women's working lives in Hungary and Austria. 1945-1995, Duke University Press, Durham.

Fodor, E., 2002. Smiling women and fighting men: the gender of the communist subject in state socialist Hungary; Gender and Society, 16 (2), 240-263.

Fodor, E., Glass, C., Kawachi, J., Popescu, L., 2002. Family policies and gender in Hungary, Poland and Romania. Communist and Post-Communists Studies, 35 (4), 475-490.

Fortino, S., 2002. La mixité au travail. La Dispute, Paris.

Glaser, B., 1968. Organizational careers. A sourcebook for theory, Aldine Publishing Company, Chicago.

Guillaume, C., Pochic, S., 2008. What would you sacrifice? Access to top management and the work/life balance. Gender Work and Organization, version online (http://www.blackwell-synergy.com/doi/abs/10.1111/j.1468-0432.2007.00354.x).

Guillaume, C., Pochic, S., 2007. La fabrication organisationnelle des dirigeants : un regard sur le plafond de verre. Travail, Genre et Sociétés 17, 79-104.

Guyonvarch, M., 2007. La flexicurité à l'épreuve du licenciement. Conflits autour d'un plan de restructurations dans un groupe pharmaceutique en France, Remest, $2,2,65-88$.

Haney, L., 2002. Inventing the needy: gender and the politics of welfare in Hungary. University of California Press.

Kanter, M. R., 1977. Men and women in the corporation, Basic Books, New York.

Karambayya, R., 1998. Caught in the crossfire: women and corporate restructuring. Canadian Journal of Administrative Sciences 15 (4), 333-338.

Laufer, J., 2005. La construction du plafond de verre : le cas des femmes cadres à potentiel. Travail et Emploi, 102, 31-44.

Laufer, J., 2004. Femmes et carrières : la question du plafond de verre. Revue Française de Gestion, 30 (151), 117-128.

Lefresne F., Sauviat C., Grimault S., 2008. Modes de gouvernance, pratiques d'anticipation et régimes de restructuration : une approche comparative, rapport IRES.

Lewis, J., 1997. Gender and welfare regimes: further thoughts. Social Politics 4 (2), 160-177. 
Lewis, J., 1992. Gender and the development of welfare regimes. Journal of European Social Policy, 2(3), 159-73.

Linhart, D., 2005. D'un monde à l'autre : la fermeture d'une entreprise. Revue de l'IRES 47(1), 81-94.

Linhart, D., 2002. Perte d'emploi, perte de soi. Eres, Paris.

Lorrain, D., 2005. La firme locale-globale: Lyonnaise des Eaux (1980-2004). Sociologie du Travail, 47, 340-361.

Martin, B., Riemens W., Wajcman J., 2000. Managerial and professional careers in an era of organizational restructuring. Journal of Sociology, 36, 3, 329-344.

Marsden, D., 1989. Marchés du travail. Limites sociales des nouvelles théories, Economica, Paris.

Maruani, M., 2003. Travail et emploi des femmes, La Découverte, Paris.

Nagy, B., 2005. The gendered management in Hungary. Perceptions and explanations. Women in Management Review 20 (5), 345-360.

Osterman, P., 1996. Broken ladders: managerial careers in the new economy, Oxford University Press, New York.

Pochic, S., 2009. Les cadres à l'épreuve de l'employabilité : passages et maintien de la frontière. In Demazière D., Gadéa C., Sociologie des groupes professionnels, La Découverte (à paraître).

Raveyre, M., 2005. Le travail dans le management des restructurations : entre déni et omission. La Revue de l'IRES, 47 (1), 95-116.

Rasmussen, B., 2001. Corporate strategy and gendered professional identities : reorganization and the struggle for recognition and positions. Gender, Work and Organization 8 (3), 291-310.

Rorive, B., 2005. Restructurations stratégiques et vulnérabilités au travail. Revue de l'IRES 47(1), 117-134.

Savage, M., 1992. Women expertise, men's authority: gendered organization and the contemporary middle classes. In: Savage, M., Witz, A. (Ed.), Gender and bureaucracy, Blackwell, Oxford, pp. 124-154.

Sayce, S., Ackers, P., Greene, A.M., 2007. Work restructuring and changing craft identity: the Tale of the Disaffected Weavers (or what happens when the rug is pulled from under your feet). Work, Employment and Society 21, p. 85-101.

Stevens, H., 2007. Destins professionnels des femmes ingénieurs. Des retournements inattendus. Sociologie du Travail 49, 443-463.

Tienari, J., 2000. Gender segregation in the making of a merger. Scandinavian Journal of Management 16, 111-144.

Vignal, C., 2005. Logiques professionnelles et logiques familiales : une articulation contrainte par la délocalisation de l'emploi. Sociologie du travail, 47 (2), 153-169.

Wajcman, J., 1998. Managing like a man. Women and men in corporate management. Polity Press, Cambridge 
Paru dans le dossier « Le plafond de verre dans les professions supérieures »

(coord. Catherine Marry, Marie Buscatto), Sociologie du travail, vol.51, n², 2009, p. 275-299.

Wajcman, J., 1996. The domestic basis of the managerial career. Sociological Review 4, 609-629.

Woodall, J., Edwards, C., Welchman, R., 1997. Organisational restructuring and the achievement of an equal opportunity structure. Gender, work and organization, 4, 1, 2-12 